\title{
Search for T-invariance violation in pd-scattering
}

\author{
Yuriy Uzikov ${ }^{1,2,3, \star}$ \\ ${ }^{1}$ Joint Institute for Nuclear Research, Dubna 141980, Russia \\ ${ }^{2}$ Dubna State University, Dubna 141980, Russia \\ ${ }^{3}$ Department of Physics, M.V. Lomonosov Moscow State University, Moscow 11991, Russia
}

\begin{abstract}
Scattering of protons with transversal polarization $P_{y}^{p}$ on deuterons with tensor polarization $P_{x z}$ provides a null-test signal for time reversal invariance violating but Pparity conserving effects. The dedicated experiment will be done at COSY facility at proton beam energy $135 \mathrm{MeV}$ using internal deuteron target. I give a brief review of results of theoretical calculations of the null-test observable within the spin-dependent Glauber theory at beam energies $100-1000 \mathrm{MeV}$ considering phenomenological T-violating Pconserving $\mathrm{NN}$-interactions.
\end{abstract}

\section{Introduction}

CP-invariance violation (or T-invariance violation under CPT-symmetry) is required to account for the baryon asymmetry of the universe [1]. Since within the standard model the CP-violation observed in physics of kaons and B-mesons is by far not sufficient to explain this asymmetry [2], other sources of CP-violation have to be found. Possible measurements are classified according to whether or not measured observables violate P-parity as well as time reversal invariance. Various efforts are undertaken for measurements of the electric dipole moments (EDM) of elementary particles and atoms which are both T-violating and P-violating. The other type of observables which are T-violating but P-conserving (TVPC) received much less attention. The reason why TVPC effects are interesting is that experimental limits on them are still quite weak, in particular considerably weaker as compared to EDM.

Integrated cross section of scattering of protons with transversal polarization $p_{y}^{p}$ on deuterons with tensor polarization $P_{x z}$ provides a null-test signal for time-reversal invariance violating but parity conserving effects [3]. Since the intensity of TVPC interactions within the standard model are extremely small [4], observation of this effect at present level of experiments would directly indicate to physics beyond the standard model. A measurement of this observable is planned at the COSY accelerator in Jülich at $135 \mathrm{MeV}$ in the TRIC experiment [5]. Theoretical study of the expected signal was performed at energies of the planned experiment [6-12]. Furthermore, certain relations between differential observables of the elastic pd-scattering caused by T-invariance requirement were obtained and degree of their violation by TVPC NN forces was studied [13, 14]. Below I will give a short review of the obtained results.

^e-mail: uzikov@jinr.ru 


\section{Null-test signal for T-invariance violation}

The total cross section of the $p d$ scattering with TVPC forces included can be written as [8]

$$
\sigma_{t o t}=\sigma_{0}^{t}+\sigma_{1}^{t} \mathbf{p}^{p} \cdot \mathbf{p}^{d}+\sigma_{2}^{t}\left(\mathbf{p}^{p} \cdot \mathbf{m}\right)\left(\mathbf{p}^{d} \cdot \mathbf{m}\right)+\sigma_{3}^{t} P_{z z}+\widetilde{\sigma} p_{y}^{p} P_{x z}^{d},
$$

where $\mathbf{p}^{p}\left(\mathbf{p}^{d}\right)$ is the vector polarization of the initial proton (deuteron) and $P_{z z}$ and $P_{x z}$ are the tensor polarizations of the deuteron. The $\mathrm{OZ}$ axis is directed along the beam direction $\mathbf{m}$, the $\mathrm{OY}$ axis is directed along the vector polarization of the proton beam $\mathbf{p}^{p}$ and the $\mathrm{OX}$ axis is chosen to form the right-hand reference frame. The integrated cross sections $\sigma_{i}^{t}(i=0,1,2,3)$ are caused by T-even Peven interaction, and the last term $\widetilde{\sigma}$ appears only in presence of the TVPC interactions and constitutes the TVPC null-test signal.

Calculation of the integrated cross sections $\sigma_{i}^{t}$ and $\widetilde{\sigma}$ at beam energies $>100 \mathrm{MeV}$ can be done on the basis of the spin-dependent Glauber theory of pd-scattering [15]. Indeed, as shown in [16], this theory allows one to describe well available data on differential spin observables at 135-200 $\mathrm{MeV}$ in forward hemisphere. In the Glauber theory one has to use the on-shell elastic NN scattering amplitudes as input. Hadronic amplitudes of the $p N$ scattering are taken in the form of [15]

$$
\begin{array}{r}
M_{N}\left(\mathbf{p}, \mathbf{q} ; \boldsymbol{\sigma}, \boldsymbol{\sigma}_{N}\right)=A_{N}+C_{N} \boldsymbol{\sigma} \hat{\mathbf{n}}+C_{N}^{\prime} \sigma_{N} \hat{\mathbf{n}}+B_{N}(\boldsymbol{\sigma} \hat{\mathbf{k}})\left(\sigma_{N} \hat{\mathbf{k}}\right)+ \\
+\left(G_{N}+H_{N}\right)(\sigma \hat{\mathbf{q}})\left(\sigma_{N} \hat{\mathbf{q}}\right)+\left(G_{N}-H_{N}\right)(\sigma \hat{\mathbf{n}})\left(\sigma_{N} \hat{\mathbf{n}}\right),
\end{array}
$$

where $\hat{\mathbf{q}}, \hat{\mathbf{k}}$ and $\hat{\mathbf{n}}$ are defined as unit vectors along the vectors $\mathbf{q}=\left(\mathbf{p}-\mathbf{p}^{\prime}\right), \mathbf{k}=\left(\mathbf{p}+\mathbf{p}^{\prime}\right)$ and $\mathbf{n}=[\mathbf{k} \times \mathbf{q}]$, respectively; $\mathbf{p}\left(\mathbf{p}^{\prime}\right)$ is the initial (final) proton momentum.

In general case TVPC NN interaction contains 18 different terms [17]. We consider here only the following terms of the t-matrix of the elastic $p N$ scattering investigated in [6]:

$$
\begin{aligned}
t_{p N}=f_{N}\left(\boldsymbol{\sigma} \cdot \boldsymbol{\sigma}_{N}\right)(\mathbf{q} \cdot \mathbf{k}) / m_{p}^{2} & +h_{N}\left[(\boldsymbol{\sigma} \cdot \mathbf{k})\left(\boldsymbol{\sigma}_{N} \cdot \mathbf{q}\right)+\left(\boldsymbol{\sigma}_{N} \cdot \mathbf{k}\right)(\boldsymbol{\sigma} \cdot \mathbf{q})-\frac{2}{3}\left(\boldsymbol{\sigma}_{N} \cdot \boldsymbol{\sigma}\right)(\mathbf{k} \cdot \mathbf{q})\right] / m_{p}^{2}+ \\
& +g_{N}\left[\boldsymbol{\sigma} \times \boldsymbol{\sigma}_{N}\right] \cdot[\mathbf{q} \times \mathbf{k}] / m_{p}^{2}+g_{N}^{\prime}\left(\boldsymbol{\sigma}-\boldsymbol{\sigma}_{N}\right) \cdot i[\mathbf{q} \times \mathbf{k}]\left[\boldsymbol{\tau} \times \boldsymbol{\tau}_{N}\right]_{z} / m_{p}^{2}
\end{aligned}
$$

Here $\sigma\left(\sigma_{N}\right)$ is the Pauli matrix acting on the spin state of the proton (nucleon $\left.N=p, n\right), \tau\left(\tau_{N}\right)$ is the isospin matrix acting on the isospin state of the proton (nucleon), the momenta $\mathbf{q}$ and $\mathbf{k}$ are defined below (2) In the framework of the phenomenological meson exchange interaction the term $g_{N}^{\prime}$ corresponds to the $\rho$-meson exchange, and the $h_{N}$-term is caused by the axial $h_{1}$-meson exchange. As shown in [18], the contribution of the $\pi$ - and $\sigma$-meson to TVPC NN interactions is excluded.

\subsection{TVPC amplitude of the forward $p d$-scattering}

One can write the forward elastic pd-scattering amplitude in a general form taking into account the TVPC interaction $[8,16]$ and then apply the generalized optical theorem to derive the formula (1) for the total cross section of the $p d$-scattering. In notations of [8] the integrated cross section $\widetilde{\sigma}$ is related to the TVPC term $\widetilde{g}$ of the forward $p d$ elastic scattering amplitude as

$$
\widetilde{\sigma}=-4 \sqrt{\pi} \operatorname{Im} \frac{2}{3} \widetilde{g}
$$

Furthermore, the forward TVPC amplitude $\widetilde{g}$ can be found within the Glauber theory [8]. Since the $g$-term gives zero contribution to $\widetilde{g}$ [8], we consider the $h$ and $g$ terms and take into account both the 
S and D wave of the deuteron. The following result was found for the forward TVPC amplitude on this way [12]

$$
\begin{array}{r}
\widetilde{g}=\frac{i}{4 \pi m_{p}} \int_{0}^{\infty} d q q^{2}\left[S_{0}^{(0)}(q)-\sqrt{8} S_{2}^{(1)}(q)-4 S_{0}^{(2)}(q)+\sqrt{2} \frac{4}{3} S_{2}^{(2)}(q)+9 S_{1}^{(2)}(q)\right] \\
{\left[-C_{n}^{\prime}(q) h_{p}+C_{p}^{\prime}(q)\left(g_{n}-h_{n}\right)\right],}
\end{array}
$$

where $S_{i}^{(j)}$ are the elastic form factors of the deuteron defined in [12]. The first term in the (big) squared brackets in $(5), S_{0}^{(0)}(q)$, corresponds to the S-wave approximation, the second term, $S_{2}^{(1)}(q)$, accounts for the S-D interference, and the last three terms contain the pure D-wave. The amplitude $\widetilde{g}$ does not contain a sum of one strong NN amplitude and one weak TVPC NN amplitude. Only products of these amplitudes appear in $\widetilde{g}$. This means that the strong (T-even P-even) background is excluded from the null-test observable $\widetilde{\sigma}$. Furthermore, available experimental uncertainties of the strong amplitudes of the NN-scattering do not affect the final result for $\widetilde{\sigma}$ drastically. That is why we believe that the accuracy of the Glauber calculations of the TVPC null-test signal is of the same order as for the pure hadronic total cross sections $\sigma_{i}^{t}(i=0,1,2,3)$.

\subsection{Why the $g^{\prime}$ term vanishes?}

It was expected that the lowest mass meson exchange allowed in the phenomenological TVPC NNinteraction, i.e. the $\rho$-meson, dominates in the null-test signal of the TVPC effects and, consequently, the strength of these effects is measured usually in units of the corresponding coupling constant $\bar{g}_{\rho}$ [19]. However, in pd scattering considered in the framework of the Glauber theory the contribution of the $g^{\prime}$-term to the null-test signal disappears. For the first time it was shown in [8]. Some qualitative explanations of the origin of this result were done in [10]. As it was shown in $[8,10]$, the crucial point is that within the Glauber theory of the multi-step scattering the highest order of rescattering is equal to $A$, where $A$ is the number of the nucleons in the target nucleus. Therefore, for pd scattering $(A=2)$ only single and double scattering mechanisms are allowed. Contributions of the higher-order rescatterings are compensated by off-shell effects in the $\mathrm{pN}$-scattering amplitudes which, therefore, are taken on-shell [20]. The single scattering contribution to the TVPC amplitude $\widetilde{g}$ is zero at zero transferred momentum $\mathbf{q}=0$, as it follows from (3). For the double scattering mechanism there are two terms which differ by the order of the pure strong and TVPC NN-scattering. For the $g^{\prime}$-type NNinteractions these two terms have opposite signs for the isospin matrix elements of the C-odd operator $\left[\tau \times \tau_{N}\right]_{z}:$

$$
<n, p\left|\left[\tau \times \tau_{N}\right]_{z}\right| p, n>=-i 2,<p, n\left|\left[\tau \times \tau_{N}\right]_{z}\right| n, p>=i 2
$$

and this fact is the second crucial point in this consideration. Indeed, the linear dependence of the $g^{\prime}$-term on $[\mathbf{q} \times \mathbf{k}]$ in (3) leads to cancellation of the strong $A_{N}$ and $B_{N}$-terms from (2) in the final formula for the TVPC charge-exchange Glauber operator of the double scattering [10]. Only the $C_{N}$ and $C_{N}^{\prime}$ strong amplitudes may contribute together with the $g^{\prime}$-term. However, the sum of $C_{n}$ and $C_{p}$ terms is identically zero [10]. Furthermore, owing to (6) the rest term in T-operator associated with the $C_{N}^{\prime}$-term is diagonal over the incoming (outcoming) proton spin states and therefore does not contribute to $\widetilde{g}$. Thus, this specific combination of isospin, spin and momentum dependencies of the $g^{\prime}$-term provides its vanishing contribution to the null-test signal in $p d$-scattering within the Glauber theory. Corrections to the Glauber theory may give a non-zero $g^{\prime}$-contribution, but as usually, such corrections are small as compared to the leading Glauber terms.

One should note that $g^{\prime}$-term gives contribution to the differential observables of the $p d$ elastic scattering and violates the relations caused by T-invariance [14]. It is assumed [8], that this interaction can give a contribution to the null-test signal if it will be included into the deuteron wave function. 


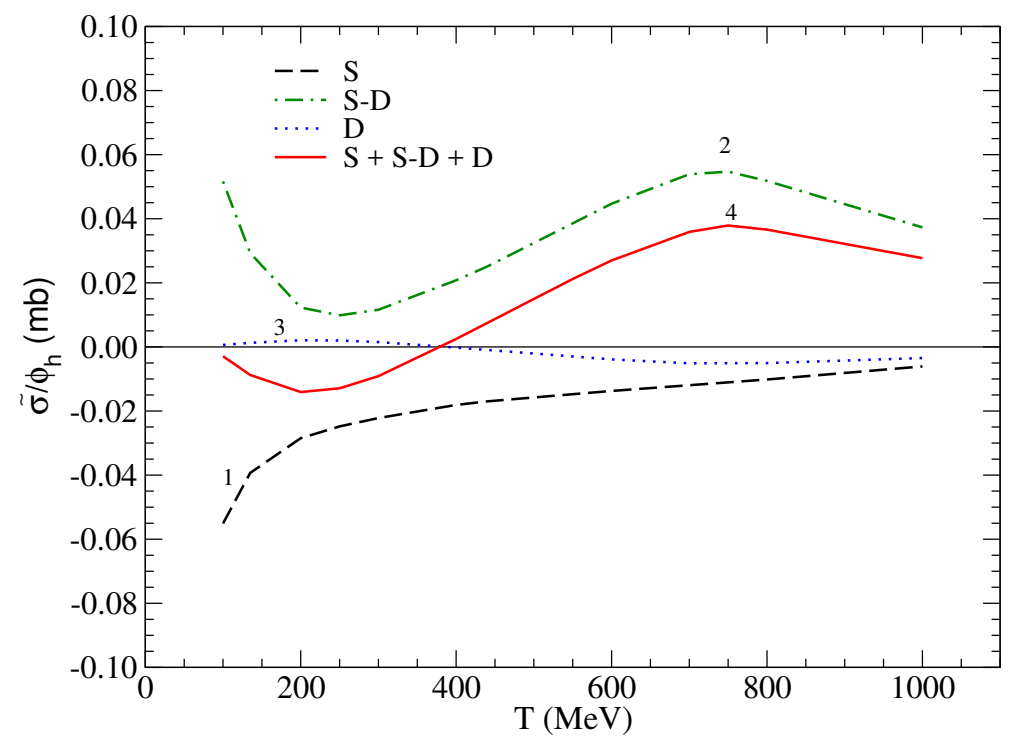

Figure 1. The TVPC signal $\widetilde{\sigma}$ for the $h$-term in units of the ratio $\left(\phi_{h}\right)$ of the TVPC and the strong $h_{1} N N$ constants versus the proton beam energy $T$. The results of calculations [12] accounting for different terms in (5) with the $\mathrm{CD}$ Bonn deuteron wave function and hadronic $\mathrm{pN}$ - amplitudes from [22] are shown by the curves: S-wave (curve 1), S-D interference (2), D-wave (3), total result (4).

\subsection{Coulomb interaction}

In the first theoretical work [6] where the null-test signal was calculated within the impulse approximation, the Coulomb interaction was not considered. In [7] the Faddeev calculations were performed only for $n d$-scattering at rather low energies $\sim 100 \mathrm{keV}$.

Straightforward calculation on the basis of the optical theorem with the Coulomb amplitude included is rather questionable. On the other hand, being a T-even P-even interaction the Coulomb $p p$ scattering cannot generate the TVPC amplitude $\widetilde{g}$, therefore its contribution to $\widetilde{g}$ is zero within the single scattering Glauber mechanism. In order to include the Coulomb interaction within the double scattering mechanism of the Glauber theory one should replace the pure hadronic T-even P-even $p p$ amplitude $M_{p}$ given in (2) by the sum $M_{p}+\tilde{f}_{p p}^{C}$, where $\tilde{f}_{p p}^{C}$ is the Coulomb $p p$-scattering amplitude. Since the Coulomb pp-amplitude consists of spin-independent term and the $\sigma \cdot \sigma_{p}$ term caused by the Fermi-statistics, it is evident that the Coulomb term is added to the spin-independent term $A_{p}$, $A_{p} \rightarrow A_{p}+\tilde{f}_{p p}^{C}$ and double spin terms $B_{p}, G_{p}, H_{p}$ but does not enter into the single spin terms $C_{p}$ and $C_{p}^{\prime}$. However, all amplitudes $A_{p}, B_{p}, G_{p}, H_{p}$ are excluded from the TVPC amplitude (5) due to the specific spin structure of the TVPC transition operator of the forward $p d$ scattering [8]. The only factor in (5) which contains the Coulomb effects is $C_{p}^{\prime}(q)$. This is because the Coulomb scattering $p p$ amplitude enters into the spin-independent term $A_{p}$, which, in turn, enters into the amplitude $C_{p}^{\prime}$ with the relativistic correction factor $q / 2 m_{p}$ [21] ( $m_{p}$ is the nucleon mass) $C_{p}^{\prime}=C_{p}+i A_{p} \frac{q}{2 m_{p}}$. When substituting this amplitude $C_{p}^{\prime}$ into (5) and making integration over the transferred momentum $q$, one can see that due to the presence of the factor $\sim q^{3}$ in the integrand the $q^{-2}$ singularity of the Coulomb amplitude at $\mathbf{q}=0$ does not lead to divergence of the $\widetilde{g}$ amplitude. As it was shown numerically [8], the Coulomb term makes rather weak influence on energy dependence of the null-test signal. 


\subsection{The deuteron D-wave contribution}

The results obtained in [8] were restricted by the deuteron S-wave component. The maximum of the null-test signal was found in this approximation at lower energies, just corresponding to the TRIC experiment (curve 1 in figure 1). In $[9,12]$ the calculations were completed taking into account the D-wave of the deuteron. It was shown for the case of the $h$ - and $g$-type of interaction that the deuteron D-wave gives very important contribution to the null-test signal due to interference between the deuteron S- and D- waves, whereas the pure D-component is negligible. First, one can see (figure 1), with the D-wave included a zero crossing of $\widetilde{\sigma}$ is possible even when the TVPC interaction itself is nonzero. In calculation [12] this occurs at beam energies about $400 \mathrm{MeV}$. Therefore, it is necessary to perform measurements at two or possibly more energies in order to achieve conclusive results. Second, according to predictions [9, 12], energies around 700-800 MeV are most promising for finding a signal (see curve 4 in figure 1). Some sensitivity to model dependent variations in the D-wave component is not too dramatic.

\subsection{Possible spurious-effect}

Let us consider possible spurious effects that could mimic a TVPC signal. One source of spurious signal is associated with a nonzero deuteron vector polarization $p_{d}^{y} \neq 0$ (in the direction of the incident-proton-beam polarization $\mathbf{p}^{p}$ ). In this case, the term $\sigma_{1} P_{y}^{p} p_{y}^{d}$ in formula (1) contributes to the asymmetry corresponding to the difference of the event counting rates for the cases of $p_{y}^{p} P_{x z}>0$ and $p_{y}^{p} P_{x z}<0$ (with the fixed sign of $P_{x z}$ ), which is planned to be measured in the TRIC experiment [5]. At the beam energy of $135 \mathrm{MeV}$, the integrated hadronic cross sections calculated in [16] on the basis of the optical theorem take the values of $\sigma_{0}=78.5 \mathrm{mb}, \sigma_{1}=3.7 \mathrm{mb}, \sigma_{2}=17.4 \mathrm{mb}, \sigma_{3}=-1.1 \mathrm{mb}$. This gives the ratio $r=\sigma_{1} / \sigma_{0}$ about 0.05 . The goal of the TRIC experiment [5] is to measure the ratio $R=\widetilde{\sigma} / \sigma_{0}$ with an error not greater than $10^{-6}$. Assuming that the signal-to-background ratio is $\widetilde{\sigma} / p_{y}^{d} \sigma_{1} \sim 10$, one can see from the result obtained for $r$ that the vector polarization of the deuteron should be smaller than $\sim 2 \times 10^{-6}$. Similar estimations can be done at $700-800 \mathrm{MeV}$. A possible way to avoid the contribution from the deuteron vector polarization is to measure the above mentioned asymmetry for opposite signs of the tensor polarization $P_{x z}$ but with the fixed direction of the vector polarization of the proton.

\section{Concluding remarks}

Theoretical study of the null-test signal in $p d$-scattering performed on the basis of the spin-dependent Glauber theory demonstrates several unexpected effects. Among them are (i) disappearance of the contribution of the lowest mass meson exchange ( $\rho$-meson) in the TVPC NN-interaction, caused by its specific isospin, spin and momentum dependence; (ii) a strong impact of the deuteron D-wave on the null-test signal arising due to destructive interference between the S- and D-wave in spite of zero transferred 3-momentum; (iii) oscillating behaviour of the null-test signal as a function of the beam energy, i.e. disappearance of the signal at some points in energy is possible even when the TVPC interaction itself is nonzero; (iv) very minor influence of the Coulomb interaction on the TVPC term of the forward scattering $p d$-amplitude $\widetilde{g}$. Only the $h$ - and $g$-type terms are accounted in (5). The remaining terms of the TVPC NN-interaction listed in [17] are either off-shell type and, therefore, do not contribute in the Glauber theory of the pd-scattering and presumably suppressed beyond this theory, or of the $h$-type. In the latter case the $h$-terms differ only in their isospin dependence but have the same spin-momentum structure. Therefore, these terms can modify (5) with regard of the relative weight of the $C_{p}^{\prime}$ and $C_{n}^{\prime}$ amplitudes which one could examine in the future. 


\section{References}

[1] A.D. Sakharov, Pis'ma v ZHETF 5, 32 (1967)

[2] A. Riotto, M. Troden, Ann. Rev. Nucl. Part. Sci 49, 35 (1999)

[3] H.E. Conzett, Phys. Rev. C 48, 423 (1993)

[4] R.S. Conti, I.B. Khriplovich, Phys. Rev. Lett. 68, 3262 (1992)

[5] COSY Proposal N 215, Spokespersons: P.D. Eversheim, B. Lorentz and Yu. Valdau, http://apps.fz-juelich.de/pax/paxwiki/images/8/8c/215-TRI_Prop_sum.pdf

[6] M. Beyer, Nucl. Phys. A 560, 895 (1993)

[7] Y.-H. Song, R. Lazauskas, V. Gudkov, Phys. Rev. C 84, 025501 (2011)

[8] Yu.N. Uzikov, A.A. Temerbayev, Phys. Rev. C 92, 014002 (2015)

[9] Yu. N. Uzikov, Jour. Phys.: Conf. Ser. 678, 012020 (2016)

[10] Yu.N. Uzikov, Eur. Phys. J.: Web of Conf. 113, 04027 (2016)

[11] Yu.N. Uzikov, A.A. Temerbayev, Int. Jour. Mod. Phys.: Conf. Series 40, 1660080 (2016)

[12] Yu.N. Uzikov, J. Haidenbauer, Phys. Rev. C 94, 035501 (2016)

[13] A.A. Temerbayev, Yu. N. Uzikov, Izv. Ross. Akad. Nauk Ser. Fiz. 79, 535 (2015).

[Bull. Russ. Acad. Sci. Phys. 79, 493 (2015)]

[14] A.A. Temerbayev, Yu. N. Uzikov, Izv. Ross. Akad. Nauk Ser. Fiz. 80, 271 (2016).

[Bull. Russ. Acad. Sci. Phys. 80(4), 242 (2016)]

[15] M.N. Platonova, V.I. Kukulin, Phys. Rev. C 81, 014004 (2010)

[16] A.A. Temerbayev, Yu.N. Uzikov, Yad. Fiz. 78, 38 (2015).

[Phys. At. Nucl. 78, 35 (2015)]

[17] P. Herczeg, Nucl. Phys. 75, 655 (1966)

[18] M. Simonius, Phys. Lett. B 78, 147 (1975)

[19] J. Engel, C.R. Gould, V. Hnizdo, Phys. Rev. Lett. 73, 3508 (1994)

[20] T.A. Osborn, Ann. Phys. 58, 417 (1970)

[21] C. Sorensen, Phys.Rev. D 19, 1444 (1979)

[22] R.A. Arndt, W.J. Briscoe, I.I. Strakovsky, R.L. Workman, Phys. Rev. C 76, 025209 (2007). http://gwdac.phys.gwu.edu 